\title{
Use of Urban Agriculture in Addressing Health Disparities and Promotion of Ecological Health in South Africa
}

\author{
Michael Rudolph ${ }^{1,}$, Evans Muchesa ${ }^{2}$, Florian Kroll ${ }^{2}$ \\ ${ }^{1}$ Office of the Vice Chancellor, University of Johannesburg, Johannesburg, South Africa \\ ${ }^{2}$ Siyakhana Growth and Development NPO, Johannesburg, South Africa
}

Email address:

michaelr@uj.ac.za (M. Rudolph), evans@siyakhana.org (E. Muchesa)

${ }^{*}$ Corresponding author

\section{To cite this article:}

Michael Rudolph, Evans Muchesa, Florian Kroll. Use of Urban Agriculture in Addressing Health Disparities and Promotion of Ecological Health in South Africa. International Journal of Natural Resource Ecology and Management. Vol. 5, No. 1, 2020, pp. $26-30$.

doi: 10.11648/j.ijnrem.20200501.14

Received: January 26, 2020; Accepted: March 10, 2020; Published: March 23, 2020

\begin{abstract}
Socio-economic disparities create conditions that contribute to poor population health. Poor health increases this socio-economic burden on the health care system and undermines society's capacity to reduce disparities and develop resilience to converging challenges regarding global, regional and national food systems. Using a structured mixed literature review design as well as both primary and secondary data sources collected through a combination of qualitative and quantitative methods and studies, this paper reflects on some key socio-economic challenges facing South Africa and shows how the findings from the research can contribute to and address public health and agricultural development policies and challenges. These themes have been presented within the broader and contemporary discourse on the relationship between ecological health, food security and national development. The agriculture system in South Africa is one of the biggest governments supported systems with a wide community reach and is thus a key sector. The debilitating effects of unhealthy communities, poor environments and socio-economic disparities are inextricably linked to food and nutrition insecurity. The findings from the reviews of the various papers and reports clearly demonstrate on the one hand the high prevalence of food and nutrition security but on the other hand reports and studies emphasize the positive outcomes of an integrated approach to addressing nutrition and food security. Furthermore, agricultural extension highlights how multi and trans-disciplinary methods based on ecological health promotion can achieve systemic changes to improve food security, public health status and also contribute socio-economic development.
\end{abstract}

Keywords: Socio-economic Disparities, Agricultural System, Food Security, Ecological Health, South Africa

\section{Introduction}

Ecological health promotion focuses on addressing and reducing inequities that may impact negatively on the health status of the disadvantaged. It also recognizes that health and well-being are a result of a combination of complex and dynamic interactions between such as social determinants, economic conditions and ecosystems. Hence, ecological health promotion transcends traditional epidemiological approaches to health in that its primary aim is to present a sustainable system of promoting, managing and maintaining health. Agriculture can play a crucial role in promoting not only agricultural productivity but can also address health disparities and promote ecological health awareness $[1,2,3]$
A key aspect of ecological health promotion is healthy nutrition which is determined by consuming safe food as part of a balanced and suitable diet that contains sufficient nutrients for optimal functioning. Thus, agriculture can facilitate the balance between good agricultural practices and health. Lack of nutrition, or malnutrition has disparaging consequences for a country's economy in that it hinders school performance, reduces individuals' income earning potential, increases the risk of disability, morbidity and mortality and contributes towards intergenerational transmission of poverty and disease. The establishment of urban and household food gardens has been recognised as a feasible way to improve nutrition, increase food access and availability, and reduce the cost of household food expenditure $[3,5,6,7]$. 
Improving people's health whilst promoting sustainable environments and more prosperous communities is one of the key challenges of the twenty-first century. Furthermore, it is an effective strategy to ease the burden on the health care system. Globally, and in many parts of South Africa, degraded ecosystems such as unsafe drinking water, poor sanitation, air and noise pollution, and neglected environments all contribute towards disease. For more favourable and sustainable health and economic outcomes, changes are required in terms of how people interact with their environments. Small scale agriculture plays a crucial role in promoting agricultural productivity, increasing food security and thereby improving health, improving urban and rural livelihoods, and acts as a driver for promoting pro-poor economic growth. Furthermore, the high prevalence of HIV/AIDS, TB and other non-communicable disease challenges that affect livelihoods and which contributes to the deterioration of the natural resource base present problems which need to be addressed $[2,7,8]$.

\section{Problem Statement}

A major challenge in SA is to reduce health disparity gaps and to generate opportunities for more people to survive childhood, promote health and well=being and thereby reach their full human potential and lead healthy and productive lives. Indeed, the National Development Plan (2012) has proposed several objectives and interventions that address these challenges. The prevailing themes of the NDP ensure equal access to health care, the promotion of healthy diets and physical exercise and addresses problems such as hunger, malnutrition and micronutrient deficiencies that affect physical growth and cognitive development. It is widely acknowledged that in order to achieve optimal health for all citizens an integrated approach must be adopted. The experience and expertise of economists, ethicists, epidemiologists, social psychologists, health promoters, public health practitioners, agriculturist, engineers, religious leaders, politicians and teachers amongst others need to be fully engaged to ensure that the full range of disciplines are represented. Adding to this list farmers and consumers must be considered in this broad equation together with relevant key stakeholders such as government, civil society and the food industry must be included or play a role. In this way, one can strive to achieve a combined understanding of health determinants and thus find solutions for sustainability [9, 10-12].

The current health interventions emphasize a model of disease and treatment in hospitals or clinical settings which are limited, isolated and compartmentalized, separating health as well as other disciplines. Furthermore, strategies and interventions are developed in isolation from other health initiatives. This has resulted, for example, in conflicting and contradictory messages being delivered to the public by the health professions as well as a lack of empirical research that directly addresses the role of media exposure to opposing/differing information on the health benefits and risks; , for example, wine, fish, and coffee consumption and even fruit and vegetable consumption and exercise. These inconsistent messages result in confusion amongst the public about what foods are best to eat which is further exacerbated in that nutrition scientists keep changing their minds [12 -14].

\section{Hypothesis}

$\mathrm{H}_{0}=$ Urban agriculture can address health disparities in vulnerable urban communities and promote ecological health in South Africa.

\section{Conceptual Framework}

The Primary Health Care (PHC) approach focuses on a more equitable, appropriate and effective response to basic health needs, as well as finding ways to solve the underlying economic, political and social determinants of ill health [2, 11]. The, health care systems have better health outcomes when built upon the PHC model, where prevention of disease and health promotion are in balance with investment $[5,8,11]$. The upsurge of chronic illness places an increased demand at both primary care and hospital levels as poorly managed and under-staffed chronic care services battle to accommodate this burden [11] Thus, raising the health status of economically disadvantaged people to the population median level of health by addressing the social and economic determinants would not only impact on overall health but would vastly improve a nation's productivity and would boost economic development Further adaptation and development of the current health system is required to address this changing profile of disease [11].

\section{Methodology}

The methodology used for this study is based on synthesis of findings from previous reports and studies carried-out Siyakhana these include; reports on Conservation Agriculture commissioned by Gauteng Department of Agriculture and Rural Development (GDARD), The NAEASF/ Edulink report, City of Johannesburg Food Security and-Prevalence study. Furthermore, there was synthesis of the literature in the areas of health; urban agriculture, food insecurity and environment health in South Africa. Open web search engine that indexes the full text or metadata of scholarly literature -Google Scholar and academic institutions repositories were also used.

\section{Findings}

Food Security Findings: The Food Security indices revealed that approximately one in five respondent households appeared severely food insecure and one in three moderately insecure. Thus, within the greater Johannesburg metropolitan area, approximately 6 million may be food insecure. About $34 \%$ of the sample were severely food access insecure [2].

To cope with food insecurity, households frequently bought 
and ate foods less preferred, bought only what was necessary, stuck to a budget, reduced portion sizes or borrowed food or money from friends or relatives. These coping mechanisms further compromised dietary quality and quantity $[2,3]$.

One in five households reported very low dietary diversity. The majority of households consumed very high starch, sugary food and drinks while fruit, vegetables and pulses were consumed less widely. This dietary profile could result in high risk of non-communicable diseases and reduced immunity which has huge health and cost ramifications [3]

Data revealed that particular socio-economic groupings including the elderly, women, the unemployed and people with low levels of education are especially vulnerable to food insecurity. This highlighted the powerful role of wider systemic and economic drivers which impacts on the food and nutritional disadvantage [6]

\subsection{Interventions}

Small-scale farming and urban agriculture

Urban agriculture provides food for the poorest of the poor. It also provides a source of income and good quality food at low cost. For middle-income families, it offers the possibility of savings and a return on their investment in urban property. For small and large entrepreneurs, it can be (or is) a profitable business. Although rapid urbanisation of peri-urban arable land is likely to lead to a decline in agricultural production with compounding factors such as limited access to water and energy, further inhibiting affordable food supply [13, 14, 25].

Nevertheless, small scale and alternative farming such as rooftop and vertical gardens as well as effective use of small spaces using innovative methods, systems and materials in available spaces such as parks schools and clinics has potential toc generate substantial food in an urban environment. Thus, executing an urban agriculture strategy could provide a solution to producing a wide range and diversity of food and thereby address food shortages and waste management whilst also delivering both health and economic benefits. Indeed, a $100 \mathrm{~m}^{2}$ plot can sustain a family for a year with fruit and vegetables yielding a nutritional intake of vitamin A, vitamin C, vitamins $B$ and iron. Furthermore, gardening has been identified as a form of exercise that can reduce the incidence of heart disease, obesity, diabetes and general therapeutic [13, 14, 25].

The potential of small-scale farming as a solution for food insecurity cannot be underestimated. With sufficient entrepreneurship skills and support, small-scale farming contributes towards the economic development and well-being of a country by providing employment and services, as well as raw materials for the agricultural and other industries. Small-scale farms also tend to be more natural and organic than commercial farms, as small-scale farmers cannot afford agrochemicals, fertilisers and genetically modified seeds. Hence, the food that is produced from the small-scale farms is healthier and contains fewer chemicals. There is also evidence that suggests that farmers who moved away from agrochemicals, genetically modified seeds and inorganic fertilisers were able to increase their food production by more than $70 \%$ per year. Small scale agriculture has the potential to break the cycle of poverty, but only if it is productive, profitable, sustainable, resilient and effectively linked to markets. To ensure that beneficiaries can achieve optimal health, agricultural projects should be nutrition-oriented promoting adequate, healthy food whilst protecting and contributing towards environmental sustainability [15].

Ecological health promotion - the process of enabling people to increase control over and to improve their health recognizes that the urban environment has significant impacts on public health [15]. Health benefits to the wider population can be achieved by facilitating the development of urban environments that promote health, equity and economic development. With a high prevalence of South Africans remaining food insecure, one of the strategies that have been used to improve the availability and access to food in South Africa's urban centres has been the concept of the community food garden. Indeed, it has been demonstrated that community food gardens can result in significant transformations of poor communities through substantially subsidizing household supplies, supplying households with an income, and increasing food diversity $[13,16]$.

The establishment gardens in schools has potential to increase awareness of the importance of good nutrition and can spread to communities. Food growing in schools had the capacity to enhance learning, skills, health and well-being outcomes, particularly in relation to diet and nutrition, for children and young people. Integrating health and nutrition education into the curriculum promotes healthy habits such as improved hygiene and sanitation, dietary diversity, nutrient preservation and informed food choices. Furthermore, ensuring that girls remain in school can prevent intergenerational transmission of malnutrition, low birth weight and child malnutrition. It is impossible to simultaneously address all the factors relating to the health disparities, but it is important to break the cycle at various key points in the systemic feedback loop which links disparities with public, environmental and socioeconomic challenges [7, $16,17]$.

\subsection{The Siyakhana Initiative}

The Siyakhana Initiative addresses critical factors such as promoting ecological health, green spaces, and raising awareness of poor nutrition, limited access to healthy food and health literacy. The Siyakhana garden has supplied produce to local Early Childhood Development Centres and NGOs that provide home-based care to HIV/AIDS patients. It has trained 30 malnourished street children in the production of mushroom farming as well as representatives from 15 NGOs and ECDC based in the inner city of Johannesburg. Siyakhana has developed innovative school programmes in order to teach both pupils and educators about growing food, about nutrition and health and to equip them with the skills to prepare healthy food. This programme has been implemented in several schools in the Gauteng province. Three hundred small-scale, community and household farmers have been trained in agro-ecological and entrepreneurial systems. It offered online 
courses for 28 staff and $\mathrm{PhD}$ students from University of Witwatersrand (Wits), Lilongwe University of Agriculture and Natural Resources and the University of Namibia; and it currently works with national, provincial and local government sectors including health, agriculture, education and economic development [13].

The Siyakhana Initiative (SI) has also conducted numerous evaluations and research studies that demonstrate the benefits of urban agricultural and nutrition interventions. In collaboration with two European and two African Universities, SI developed a pilot online course in Food Security and generated a comprehensive report on women in agriculture NAEAFS ACP Edulink 2016; conducted a nationwide evaluation of nineteen food garden projects; established a rooftop garden in Kotze street, Braamfontein; trained school children in urban agricultural practice and nutrition; and provided health promotion courses for community, civil society and government [14].

At policy level cities or local governments need to have food systems governance for resilience. According to the City of Johannesburg Food Resilience Programme Evaluation carried out by Siyakhana, fundamentally, it is to re-orient the strategy and policy towards an adaptive urban food systems governance approach which moves away from the top-down implementation of discrete projects farmed out via municipal tendering processes or starter pack and food package distribution programmes [14]

\section{Conclusion}

Economic growth is crucial for health. Investment in health and its determinants is an important strategy to boost economic development. The need for inter- and multi-disciplinary approach to bridge the disparities gap and to effectively address the multiple determinants of South Africa's health disparities is clearly shown and identified. Agricultural extension, because of its extensive network can effectively help to address some of the disparities in health and ecological health. South African can exploit and build on the extensiveness network of the public extension system to address health and nutrition issues. Farmers can learn how to grow a wide diversity of nutritious crops within available budgets and applying biodiversity methods. Programmes and policies should target and encompass all key sectors of government and society and should propose interventions that could measurably improve people's environments and conditions of daily life $[17,19,20]$.

There is need of consolidated efforts from local and national governments need to ensure that the urban and inner-city environments are healthier for their citizens. This should include allocating funding towards research on the nutritional status of urban populations, the zoning of specific land for the use of urban agriculture and ensuring co-operation between all role players in managing and implementing nutrition and urban agricultural projects. Nutrition interventions that provide food insecure individuals with a diversity of fresh fruits and vegetables will vastly improve micronutrient status - especially in women and children - and will reduce levels of stunting and wasting. Incorporating food relief into treatment protocols for patients co-infected with $\mathrm{TB}$ and HIV, as well as chronic NCDs, will enhance drug efficacy and enable patients to tolerate drug interactions more effectively. Similarly, supplementation with highly bio-available micronutrients will support conventional drug-based treatments $[8,17,19,23]$.

There is need of establishing protocols to ensure gender equity will address the health disparities to which women are subjected. Women tend to suffer greater levels of malnutrition and violence and still have to bear the burden of earning an income whilst caring for their children and the elderly. Better environmental management - such as cleaner drinking water, improved sanitation and providing household energy - can improve the health and lives of women. Furthermore, healthier and productive ecosystems provide income-generating opportunities for women and additional resources for the household. Enhanced environmental conditions improve a child's survival rate, thus relieving women of some of the childcare burdens. Hence, formal policy processes and programmes need to be engaged to achieve sustainable change and gender equity $[21,22]$.

South Africa's problem of burgeoning epidemics of chronic illness and high maternal, neonatal and childhood mortality, makes it imperative to prioritise is given to primary prevention through school-based health promotion and nutrition interventions. Learning opportunities for both schools and communities whereby ecological health promotion is used as a tool to promote health and prevent disease should include nutrition workshops, growing food and greening initiatives, environmental awareness, and entrepreneurship and ownership. Effective and efficient use of human and material resources can lead to new ways of thinking about nutrition and can cultivate a culture of excellent leadership and entrepreneurship [8, 17, 22].

Improving health and nutrition is not just about investing more. Equally important is conducting sound policy analysis, ensuring that these policies are linked to appropriate action, and developing the capacity to manage effective nutrition programmes. Strengthening commitment to confronting the crisis of malnutrition and forging new partnerships is critical to making a tangible and measurable impact. These partnerships must include government, communities and the NGO sector, as well as the corporate sector, whose role in fortifying food and assuming responsibility for the nutritional content of snacks and fast food is crucial [24].

Putting an end to extreme malnutrition can lay the foundations for improving the health and wellbeing of the present generations and lead to benefits for future ones, should also be at the core of public extension. Embedded in broader systemic shifts towards food security, food gardens and fortified foods can become sound and sustainable policies for good nutrition which are essential for poverty reduction. The Siyakhana Initiative has made impressive progress to bring a broader awareness of the challenges of food security, nutrition and its links with health and sustainable development [17]. 
The agriculture system is one of the biggest governments supported system in South Africa and has a wide community reach. The harmful effects of unhealthy communities and poor environments and socio-economic disparities can also be linked to food and nutrition insecurity $[17,23]$.

\section{References}

[1] Badland, Hannah, and Jamie Pearce. "Liveable for whom? Prospects of urban liveability to address health inequities." Social Science \& Medicine (2019).

[2] Ahonen, E. Q., Fujishiro, K., Cunningham, T., \& Flynn, M. (2018). Work as an inclusive part of population health inequities research and prevention. American journal of public health, 108 (3), 306-311.

[3] Pereira, L., \& Drimie, S. (2016). Governance arrangements for the future food system: Addressing complexity in South Africa. Environment: Science and Policy for Sustainable Development, 58 (4), 18-31.

[4] Wilde, P. (2018). Food policy in the United States: An introduction. Routledge.

[5] Pugh, S., Merten-Lentz, K., Buttriss, J., Pravst, I., Hieke, S., Mills, C.,... \& Corvalán, C. (2019). Health Claims and Food Labelling. Royal Society of Chemistry.

[6] Barry, M. M.(2019). Reframing the Challenge of Promoting Population Mental Health. In Implementing Mental Health Promotion (pp. 35-57). Springer, Cham.

[7] van Vuuren, D., Lucas, P., Calvin, K. V., Chung, S. H., Harfoot M., Hedden, S.,... \& Hughes, B. B. (2019). Pathways towards Sustainable Development-Global Environment Outlook (GEO-6): Healthy Planet, Healthy People Chapter 22. Global Environment Outlook (GEO-6): Healthy Planet, Healthy People.

[8] Mosia, S. J. (2018). Students' perspectives on the primary health care practice learning enviroment (Doctoral dissertation, University of the Free State).

[9] Cumming, T. L., Shackleton, R. T., Förster, J., Dini, J., Khan, A., Gumula, M., \& Kubiszewski, I. (2017). Achieving the national development agenda and the Sustainable Development Goals (SDGs) through investment in ecological infrastructure: A case study of South Africa. Ecosystem services, 27, 253-260.

[10] Patierno, S., \& Sitkin, S. B. (Eds.). (2019). Behavioral Science $\&$ Policy, Volume 4 (No. 1). Brookings Institution Press.

[11] Gualandris, J., \& Klassen, R. D. (2018). EMERGING DISCOURSE INCUBATOR: Delivering Transformational Change: Aligning Supply Chains and Stakeholders in Non Governmental Organizations. Journal of Supply Chain Management, 54 (2), 34-48.

[12] Crane, A., Matten, D., \& Spence, L. (Eds.). (2019). Corporate social responsibility: Readings and cases in a global context. Routledge.
[13] Kroll, F. J., Rudolph, M. J., Simatele, D. (2015). Gauteng City Region Observatory Food Systems Review Paper (Working Paper Series). Food Security Working Paper 1: A systemic review of food security in the Gauteng City Region.

[14] Kroll, F. J., Rudolph, M. J. (2016). City of Johannesburg Food Resilience Programme Evaluation: Final Report. Wits School of Geography, Archaeology and Environmental Studies (GAES), Wits Siyakhana Initiative and Wits Commercial Enterprise.

[15] Franz, C., \& Ghebreyesus, T. A. (2019). The Road to Universal Health Coverage: Innovation, Equity, and the New Health Economy. JHU Press.

[16] Rizzo, A., Sandberg, M., Johansson, T., \& Wenngren, J. (2018) Food on the Roof: Developing an IT platform to visualize and identify suitable locations for roof farming in cold climates.

[17] Rudolph M, Kroll F, Beery M, Marinda E, Sobiecki J, Douglas G, (2013). A pilot study assessing the impact of a fortified supplementary food on the health and well-being of crèche children and adult TB patients in South Africa. PLoS One. 2013; 8 (1): e55544.

[18] Rogan, M. (2018). Food poverty, hunger and household production in rural Eastern Cape households. Development Southern Africa, 35 (1), 90-104.

[19] Saha, M. (2019). Mainstreaming Nutrition into Agricultural Extension: Two Projects that Integrated Agricultural Intervention.

[20] Hunter, D., Borelli, T., Olsen Lauridsen, N., Gee, E., Rota Nodari, G., Moura de Oliveira Beltrame, D.,... \& Güner, B. (2018). Biodiversity mainstreaming for healthy \& sustainable food systems: A toolkit to support incorporating biodiversity into policies and programmes.

[21] Gostin, L. O., Monahan, J. T., Kaldor, J., Debartolo, M., Friedman, E. A., Gottschalk, K.,... \& Cabal, L. (2019). The Lancet Commissions The legal determinants of health: harnessing the power of law for global health and sustainable development.

[22] Ndirangu, M., \& Sztam, K. A. (2015). HIV and Nutrition. In Preventive Nutrition (pp. 645-675). Springer, Cham.

[23] Quan, R. J. D. (2019). Human rights and the gender dynamics of climate change. In Elgar Encyclopedia of Environmental Law (pp. 235-253). Edward Elgar Publishing Limited.

[24] Solomon, S. (2019). Understanding the Impacts of Climate Change on Water Access and the Lives of Women in Tharparkar District, Sindh Province, Pakistan: A Literature Review, 1990-2018.

[25] Nogeire-McRae, T., Ryan, E. P., Jablonski, B. B., Carolan, M., Arathi, H. S., Brown, C. S.,... \& Schipanski, M. E. (2018). The role of urban agriculture in a secure, healthy, and sustainable food system. BioScience, 68 (10), 748-759. 